\title{
Hepatitis E Virus Seroprevalence Among Liver Transplant Recipients with Persistent Elevation of Liver Enzymes: A Single Center Report
}

\author{
${ }^{1}$ Gastroenterohepatology Research Center, Shiraz University of Medical Sciences, Shiraz, Iran \\ ${ }^{2}$ Health Policy Research Center, Shiraz University of Medical Sciences, Shiraz, Iran \\ ${ }^{3}$ Shiraz Transplant Research Center, Shiraz University of Medical Sciences, Shiraz, Iran \\ ${ }^{4}$ Division of Gastroenterology, Toronto General Hospital, University of Toronto, Toronto, Canada
}

Maryam Chorami, MD¹; Kamran Bagheri Lankarani, MD²; Fardad Ejtehadi, MD'; Seyed Ali Malek-Hosseini, MD³; Maryam Moini, MD,4*

\begin{abstract}
Background: Chronic hepatitis E infection has been reported in solid organ transplant recipients following acute hepatitis due to the compromised immune status. Almost all reports are from areas where hepatitis E virus (HEV) genotypes 3 and 4 are the dominant genotypes. This study was conducted to investigate the role of hepatitis $\mathrm{E}$ infection as an etiology for liver enzymes elevation in liver transplant recipients from the largest liver transplant program in Iran.

Methods: In a prospective study from June to December 2015, in a single liver transplantation center in Iran, all adult liver recipients who were investigated for the etiology of persistent elevation of liver enzymes were tested for HEV serology status. Results: Of 122 patients included in the study, 19 (15.6\%) were positive for HEV serology. Seropositive patients were significantly older than seronegative ones (mean age 43.79 vs. 31.58, $P<0.001$ ); however, they were not different in other characteristics including sex distribution and mean of liver enzymes in each occasion. Liver biopsies were done in 16 HEV seropositive patients and none of the biopsies showed evidence for acute or chronic viral hepatitis.

Conclusion: In this study, with $15.6 \%$ rate of HEV seropositivity in liver recipients with persistent elevation of liver enzymes, we were not able to confirm any clinical evidence for active acute or chronic hepatitis $\mathrm{E}$ infection. This could theoretically be attributed to the fact that the dominant prevalent HEV genotype in our endemic area is not associated with a chronic form of infection.

Keywords: Chronic Hepatitis E, Liver transplantation

Cite this article as: Chorami M, Lankarani KB, Ejtehadi F, Malek-Hosseini SA, Moini M. Hepatitis E virus seroprevalence among liver transplant recipients with persistent elevation of liver enzymes: a single center report. Arch Iran Med. 2021;24(1):22-26. doi: 10.34172/aim.2021.04.
\end{abstract}

Received: February 11, 2020, Accepted: October 20, 2020, ePublished: January 1, 2021

\section{Introduction}

Liver transplantation is the treatment of choice for patients with end-stage liver disease and could be a lifesaving option in acute liver failure. ${ }^{1}$ It is also considered as a curative treatment for a number of inherited metabolic disorders and selected cases of malignancies involving the liver. The posttransplant management of liver recipients is challenging from different aspects including the need for prolonged immunosuppression and its related complications. Long-term immunosuppressive therapy harbors risks of various complications including increased risk of viral, bacterial and fungal infections, ${ }^{2}$ and infectious complications are among the long list of differential diagnoses of persistent elevation of liver enzymes in liver recipients. The hepatitis $\mathrm{E}$ virus (HEV) infection, previously considered as causing only acute hepatitis, has been reported to be capable of evolving to a chronic form in recipients of solid organ transplantation, ${ }^{3}$ as well as in other immunocompromised states as hematologic patients receiving chemotherapy, ${ }^{4}$ and human immunodeficiency virus (HIV)-infected patients. ${ }^{5}$

Studies representing prevalence data have revealed variable frequencies of $\mathrm{HEV}$ infection in liver transplant recipients ranging from $3 \%$ to $27.5 \%$ in different European and North American countries. ${ }^{6-8}$

There are few published epidemiologic studies on the seropositive prevalence of hepatitis $\mathrm{E}$ in general and different patient populations in Iran, ${ }^{9,10}$ but less is known about the HEV infection among recipients of solid organ transplantation.

The present study is a prospective single center study, designed to investigate HEV serology status and possible role of $\mathrm{HEV}$ infection in persistent liver enzyme elevation in liver transplant recipients who were transplanted in Shiraz Organ Transplantation Center.

\section{Patients and Methods}

Study Subjects

This was a prospective, cross-sectional study in a population of adult (18 years and above) liver transplant recipients 
with persistent elevation of liver enzymes who were transplanted in Shiraz Organ Transplant Center, Nemazi Hospital, Shiraz University of Medical Sciences. The study was done in both inpatient and outpatient settings within 6 months from June to December 2015. Persistent elevation of liver enzymes was defined as elevation in alanine transaminase (ALT), Aspartate transaminase (AST) or alkaline phosphatase (ALP) to more than 1.5 times upper limit of normal in two consecutive measurements, at least two weeks apart. Recipients within the first post-transplant month were not included in the study. For every patient, an extensive workup was done to investigate the cause of elevated liver enzymes. These included various laboratory and imaging studies and, in some cases, liver biopsy. Viral serology testing for hepatitis C (HCV), hepatitis B (HBV), hepatitis A (HAV) and cytomegalovirus (CMV) infections were among these investigations. In addition, all patients were tested for the presence of anti-HEV IgG and IgM antibodies (Ab). This was done using ELISA kit (HEV IgG IgM Antibody ELISA, Dia.Pro, Milano, Italy) according to the manufacturer's instructions. ${ }^{11}$

\section{Statistical Analysis}

The obtained data was analyzed using Statistical Package for Social Sciences (SPSS), released 2012 (IBM SPSS Statistics for Windows, version 21.0. Armonk, NY: IBM Corp). Frequencies and percentages were calculated. We used the non-parametric Mann-Whitney U test for comparing quantitative variables as age, ALT, AST and ALP between the two groups of patients. The Pearson chisquare test was performed for comparing the frequencies of categorial variables between the two groups. $P$ values $<$ 0.05 were considered statistically significant.

\section{Results}

A total of 122 including 77 male (63.1\%) and 45 (36.9\%) female patients with a mean age of $33.48 \pm 12.41(\mathrm{SD})$ years were enrolled in the study. The most common etiology of liver disease leading to transplantation was autoimmune-related liver diseases (39.3\%) followed by cryptogenic cirrhosis $(23.8 \%)$ and Wilson disease (9\%) (Table 1).

Nineteen patients out of 122 (15.6\%) were seropositive for total HEV Ab including 4 patients who were positive for both HEV IgG and IgM Ab. The prevalence of HEV seropositivity was slightly higher among male patients (16.9\% vs. $13.3 \%)$ but with no statistically significant difference (odds ratio: $1.32, P=0.6,95 \%$ CI: 0.46-3.76). Liver biopsies were done in 16 patients with positive total $\mathrm{HEV} \mathrm{Ab}$ including 3 patients with positive $\mathrm{HEV}$ IgM Ab. Among them, seven biopsy results were normal. Acute rejection was reported in four, chronic rejection in two and steatosis in three patients. In the three patients who had positive HEV IgM serology and underwent liver biopsy, one showed histologic evidence for acute rejection,
Table 1. Characteristics of the 122 Liver Recipients with Persistent Elevation of Liver Enzymes

\begin{tabular}{lc}
\hline Variable & Data \\
\hline Male, sex & $77(63.1 \%)$ \\
Age $(y)$, Mean \pm SD & $33.48 \pm 12.41$ \\
Etiology of liver disease & \\
Autoimmune & $48(39.3 \%)$ \\
Cryptogenic & $29(23.8 \%)$ \\
Inherited metabolic disorders & $16(13.1 \%)$ \\
Viral hepatitis & $14(11.5 \%)$ \\
Budd Chiari syndrome & $3(2.5 \%)$ \\
Nonalcoholic steatohepatitis & $2(1.6 \%)$ \\
Hepatocellular carcinoma & $2(1.6 \%)$ \\
Others & $8(6.6 \%)$ \\
Liver biopsy results & \\
Normal & $38(31.1 \%)$ \\
Acute rejection & $35(28.7 \%)$ \\
Steatosis & $19(15.6 \%)$ \\
Chronic rejection & $10(8.2 \%)$ \\
Biliary complications & $5(4.1 \%)$ \\
Total number of biopsies & $107(87.7 \%)$ \\
\hline
\end{tabular}

$\mathrm{SD}$, standard deviation.

one chronic rejection and one had a normal liver biopsy. None of the 16 patients with positive total HEV Ab had histologic changes in favor of acute or chronic viral hepatitis. In HEV seropositive patients, the median ALT level in the first occasion was $53.0 \mathrm{IU} / \mathrm{mL}$ with first quartile (Q1) of 31.0 and third quartile (Q3) of $98.0 \mathrm{IU} / \mathrm{mL}$. On second occasion, the median ALT level was $76.0 \mathrm{IU} / \mathrm{mL}$ with Q1 of 41.0 and Q3 of 101.0 IU/mL. Maximum ALT levels were $341 \mathrm{IU} / \mathrm{mL}$ and $244 \mathrm{IU} / \mathrm{mL}$ on each occasion.

All the patients received appropriate treatment for the driving etiologies of elevated liver enzymes with subsequent improvement. Acute rejection was treated with steroid pulses and for chronic rejection, immunosuppression treatment was intensified. Biliary strictures were treated with radiologic or endoscopic intervention. CMV infection, which was detected in one patient, was improved after a complete course of antiviral treatment with ganciclovir. None of the patients were diagnosed with acute viral hepatitis $\mathrm{A}, \mathrm{B}$ or $\mathrm{C}$ and none of the patients with chronic viral hepatitis as their primary liver disease showed evidence of recurrence. One patient died due to severe variceal bleeding not responding to interventional treatment during hospital admission. Of those patients with positive HEV serology, no one received antiviral treatment.

Patients with positive $\mathrm{HEV} \mathrm{Ab}$ were older than seronegative patients with a significant difference in mean age $(P<0.001)$. However, there was no difference in sex distribution between the two groups, and the means of ALT, AST and ALP in each consequence were not different in HEV seropositive patients from seronegative ones (Table 2). 
Table 2. Comparison of the Characteristics of Liver Recipients with Persistent Elevation of Liver Enzymes Categorized Into 2 Groups Based on Hepatitis E Serology

\begin{tabular}{lcc}
\hline Variables & HEV Seropositive Liver Recipients & HEV Seronegative Liver Recipients \\
\hline Age (years) mean \pm SD & $43.79 \pm 10.73$ & $31.58 \pm 11.79$ \\
Male sex (\%) & $13(68.4 \%)$ & $64(62.1 \%)$ \\
Liver enzymes Occasion 1 & & $<0.001^{*}$ \\
ALT (IU/mL); Median, Q1, Q3 & $53.0,31.0,98.0$ & $45.0,26.0,69.5$ \\
AST (IU/mL); Median, Q1, Q3 & $86.0,29.0,121.0$ & $79.0,35.5,113.0$ \\
ALP (IU/mL); Median, Q1, Q3 & $285.0,215.0,376.0$ & $345.5,211.75,551.0$ \\
Liver enzymes Occasion 2 & & $0.278^{*}$ \\
ALT (IU/mL); Median, Q1, Q3 & $76.0,41.0,101.0$ & $45,0,26.0,86.0$ \\
AST (IU/mL); Median, Q1, Q3 & $97.0,73.0,132.0$ & $81.0,37.5,130.0$ \\
ALP (IU/mL); Median, Q1, Q3 & $342.0,256.0,603.0$ & $0.351^{*}$ \\
\hline
\end{tabular}

ALP, Alkaline phosphatase; ALT, Alanine aminotransferase; AST, Aspartate aminotransferase; HEV, hepatitis E virus; Q1, quartile 1; Q3, quartile 3; SD, standard deviation.

* $P$ values calculated using Mann-Whitney $U$ test.

** $P$ value calculated using Pearson chi-square test.

\section{Discussion}

The hepatitis E infection was mostly known for the acute form of viral hepatitis with a clinical presentation similar to acute hepatitis A, usually self-limited but associated with high mortality among pregnant women. ${ }^{12}$ The main rout of transmission is fecal-oral and waterborne infection is responsible for most of outbreaks in developing countries. Other modes of transmission include: zoonotic, foodborne, blood borne and perinatal transmission. ${ }^{13}$

Genotypes 1 and 2 are transmitted fecal-orally, restricted to humans and seen with the outbreaks or sporadic cases of acute hepatitis E in developing countries. ${ }^{12}$ Genotypes 3 and 4 are seen in both animals and humans in North America, Europe and Asia. Genotype 3 can cause sporadic cases of milder form of acute hepatitis E. ${ }^{14}$ Most chronic cases of hepatitis E are related to genotype 3 and some to genotype 4; however, a few cases of chronic hepatitis from HEV genotype 1 have also been reported. ${ }^{15,16}$ Swine, deer, wild boar and rabbit are the zoonotic hosts for genotype 3 and, swine and boar for genotype 4. Transmission to humans occurs through undercooked meat of these animals. ${ }^{12}$ The seroprevalence of anti-HEV antibody varies among difference countries. Rates between 5\% to 30\% have been reported from developing countries while in the United States and Western Europe, rates of 3\% and less have been reported. ${ }^{6}$

The chronic hepatitis $\mathrm{E}$ infection in solid organ transplant recipients was first reported by Kamar et al. ${ }^{17}$ They described 14 cases of acute hepatitis $E$ in recipients of different solid organs; of those, 8 cases developed chronic hepatitis. The authors suggested that in the presence of immunosuppression, acute hepatitis E may evolve to a chronic infection. The risk of developing chronic infection following acute hepatitis $\mathrm{E}$ is estimated to be more than $60 \%$ for all types of solid organ transplants. ${ }^{6,18}$

The geographic variation in the prevalence of different hepatitis $\mathrm{E}$ genotypes may be a determinant factor in the incidence and prevalence of chronic hepatitis $\mathrm{E}$ infection among solid organ recipients. Based on the current study, seroprevalence of HEV Ab positivity was $15.6 \%$ among our patient population of 122 liver recipients with persistent elevation of liver enzymes. Epidemiologic studies have reported very variable rates for HEV seroprevalence in Iran, from $1.1 \%$ to $46 \%$ based on the population, agegroup and geographic area of the study. ${ }^{19,20}$ In a systematic review and meta-analysis on seroprevalence of hepatitis E in Iran by Behzadifar et al, strong heterogenicity was observed among the epidemiologic reports and the pooled HEV seroprevalence was estimated at $10 \%{ }^{21}$ There are few reports of $\mathrm{HEV}$ seroprevalence in transplant population of patients in Iran and these are mainly from kidney transplant recipients. Rostamzadeh Khameneh et al reported 30.8\% HEV IgG positivity in a population of 91 kidney transplant recipients with no significant correlation with ALT elevation in these patients. ${ }^{22}$ In another study by Zeraati et al, HEV IgG seropositivity was reported at $20.9 \%$ among 110 recipients of kidney transplant with no significant difference in liver and renal function compared to seronegative patients. ${ }^{23}$ In the present study, HEV IgG positivity among liver transplant recipients with persistent elevation of liver enzymes was detected as $15.6 \%$. Even though this selected study population is not a sample representing all liver transplant recipients, the rate of HEV seropositivity was less than the reports from kidney transplant patients in Iran but mildly higher than the estimated rate for the general population.

In our study, liver transplant recipients with $\mathrm{HEV} \mathrm{Ab}$ positive serology showed modest elevation in ALT and AST - not a picture compatible with acute viral hepatitis. The same was true for those with a positive HEV IgM antibody. Also, there were no histology changes suggestive of acute or chronic viral hepatitis in seropositive patients who underwent liver biopsies. Based on these observations, we can conclude that the positive HEV serology in our patients is mostly related to the previous exposure to the virus rather than an active acute or chronic viral hepatitis. 
Not enough data is available on the dominant HEV genotype in Iran. However, based on the epidemiologic studies reporting genotype 1 and 2 as the main prevalent genotypes in developing countries and genotype 1 mainly in Asia, ${ }^{6,24}$ most cases of the hepatitis $E$ infection in Iran are probably related to genotype $1 \mathrm{HEV}$ infection with a fecaloral rout of transmission. Whereas epidemic outbreaks of acute hepatitis $\mathrm{E}$ have not been reported in Iran, most HEV seropositive cases are presumed to be due to sporadic exposures to the virus with mild symptoms not diagnosed at the time of infection - a pattern similar to hepatitis A infection. The fact that HEV seropositive patients were older than seronegative ones in our study may also points to the relation between the increased chance of exposure to the potential sources of infection with increasing age.

On the other hand, genotypes 3 and 4 are less likely to be the cause of HEV infection in Iran as their rout of transmission is from uncooked meat of certain animals which are not used in Iran, mostly due to religious prohibition or unpopularity.

This study was the first report on HEV seroprevalence in a population of liver transplant recipients in Iran who were investigated for the competing causes of elevated liver enzymes. We detected a seropositivity rate of $15.6 \%$ with no evidence for acute or chronic viral hepatitis in our patients. Based on the findings from this study, we can conclude that HEV seropositivity in our liver transplant population was not associated with evidence of chronic viral hepatitis and most probably represented a remote viral exposure. Lack of evidence for the chronic hepatitis $E$ infection in our immunocompromised population of patients might be due to the different virus epidemiology and the dominant prevalent genotype which is most probably genotype 1 . The main limitation of this study was the lack of molecular testing for the presence of HEV RNA due to the limited resources. Absence of a control group for comparing the HEV seroprevalence could also be considered a limitation. More studies with larger numbers of patients with variable transplanted organs and, using molecular testing for virus RNA and genotyping may be required to better address the true prevalence of HEVrelated chronic hepatitis in this population of patients in Iran.

\section{Authors' Contribution}

$M M$ and $K B L$ designed the work. MC, MM, KBL, FE and SAM were involved in clinical work and patient's recruitment. MC did data gathering. MM did data analysis. MC and MM wrote the paper.

\section{Conflict of Interest Disclosures}

The authors declare that there are no conflicts of interest.

\section{Ethical Statement}

The study was reviewed and approved by the Ethical Committee of Shiraz University of Medical Sciences. Written informed consents were obtained from all participants.

\section{References}

1. Saidi RF, Kazemaini SM, Malekzadeh R. Current Challenges of Liver Transplantation in Iran. Middle East J Dig Dis. 2018;10(1):45-49. doi: 10.15171/mejdd.2017.90.

2. Lucey MR, Terrault N, Ojo L, Hay JE, Neuberger J, Blumberg E, Teperman LW. Long-term management of the successful adult liver transplant: 2012 practice guideline by the American Association for the Study of Liver Diseases and the American Society of Transplantation. Liver Transpl. 2013;19(1):3-26. doi: 10.1002/lt.23566.

3. Buffaz C, Scholtes C, Dron AG, Chevallier-Queyron P, Ritter J, Andre $\mathrm{P}$, et al. Hepatitis $\mathrm{E}$ in liver transplant recipients in the Rhone-Alpes region in France. Eur J Clin Microbiol Infect Dis. 2014;33(6):1037-43. doi: 10.1007/s10096-013-2042-2.

4. Ollier L, Tieulie N, Sanderson F, Heudier P, Giordanengo V, Fuzibet JG, et al. Chronic hepatitis after hepatitis E virus infection in a patient with non-Hodgkin lymphoma taking rituximab. Ann Intern Med. 2009;150(6):430-1. doi: 10.7326/0003-4819-150-6-200903170-00026.

5. Dalton HR, Bendall RP, Keane FE, Tedder RS, ljaz S. Persistent carriage of hepatitis E virus in patients with HIV infection. N Engl J Med. 2009;361(10):1025-7. doi: 10.1056/ NEJMc0903778.

6. Zhou X, de Man RA, de Knegt RJ, Metselaar HJ, Peppelenbosch MP, Pan Q. Epidemiology and management of chronic hepatitis E infection in solid organ transplantation: a comprehensive literature review. Rev Med Virol. 2013;23(5): 295-304. doi: 10.1002/rmv.1751.

7. Haagsma EB, Niesters HG, van den Berg AP, RiezebosBrilman A, Porte RJ, Vennema $\mathrm{H}$, et al. Prevalence of hepatitis $\mathrm{E}$ virus infection in liver transplant recipients. Liver Transpl. 2009;15(10):1225-8. doi: 10.1002/lt.21819.

8. Halac U, Beland K, Lapierre P, Patey N, Ward P, Brassard $\mathrm{J}$, et al. Chronic hepatitis E infection in children with liver transplantation. Gut. 2012;61(4):597-603. doi: 10.1136/ gutjnl-2011-300708.

9. Taherkhani R, Farshadpour F, Epidemiology of hepatitis E virus in Iran. World J Gastroenterol. 2016;22(22):5143-53. doi: 10.3748/wjg.v22.i22.5143.

10. Farshadpour F, Taherkhani R, Ravanbod MR, Eghbali SS, Taherkhani S, Mahdavi E. Prevalence, risk factors and molecular evaluation of hepatitis E virus infection among pregnant women resident in the northern shores of Persian Gulf, Iran. PLoS One. 2018;13(1):e0191090. doi: 10.1371/ journal.pone.0191090.

11. Third generation Enzyme Immunoassay for the determination of $\lg$ antibodies to Hepatitis E Virus in human serum and plasma for "in vitro" diagnostic use only. In: Diagnostic Bioprobes Srl DIA.PRO, ed. Milano; Italy: DIA.PRO; 2009.

12. Nimgaonkar I, Ding Q, Schwartz RE, Ploss A. Hepatitis E virus: advances and challenges. Nat Rev Gastroenterol Hepatol. 2018;15(2):96-110. doi: 10.1038/nrgastro.2017.150.

13. Mushahwar IK, Hepatitis E virus: molecular virology, clinical features, diagnosis, transmission, epidemiology, and prevention. J Med Virol. 2008;80(4):646-58. doi: 10.1002/ jmv.21116.

14. Fang SY, Han H. Hepatitis E viral infection in solid organ transplant patients. Curr Opin Organ Transplant. 2017;22(4):351-5. doi: 10.1097/mot.0000000000000432.

15. Robins AEM, Bowden DJ, Gelson WTH. Chronic genotype 1 hepatitis $\mathrm{E}$ infection from immunosuppression for ileo-colonic Crohn's disease. Oxf Med Case Reports. 2018;2018(9):omy059. doi: 10.1093/omcr/omy059.

16. Singh A, Seth R, Gupta A, Shalimar, Nayak B, Acharya $\mathrm{SK}$, et al. Chronic hepatitis E - an emerging disease in an immunocompromised host. Gastroenterol Rep (Oxf). 2018;6(2):152-5. doi: 10.1093/gastro/gow024.

17. Kamar N, Selves J, Mansuy JM, Ouezzani L, Peron JM, Guitard $\mathrm{J}$, et al. Hepatitis E virus and chronic hepatitis in organtransplant recipients. N Engl J Med. 2008;358(8):811-7. doi: 
10.1056/NEJMoa0706992.

18. Kamar N, Garrouste C, Haagsma EB, Garrigue V, Pischke S, Chauvet $\mathrm{C}$, et al. Factors associated with chronic hepatitis in patients with hepatitis $\mathrm{E}$ virus infection who have received solid organ transplants. Gastroenterology. 2011;140(5):14819. doi: 10.1053/j.gastro.2011.02.050.

19. Farshadpour F, Taherkhani R, Makvandi M. Prevalence of hepatitis E virus among adults in south-west of Iran. Hepat Res Treat. 2015;2015:759589. doi: 10.1155/2015/759589.

20. Ghorbani GA, Alavian S-M, Esfahani AA, Asaari S. Seroepidemiology of hepatitis E virus in Iranian soldiers; 2007.

21. Behzadifar M, Lankarani KB, Abdi S, Taheri Mirghaed M, Beyranvand G, Keshavarzi A, et al. Seroprevalence of hepatitis
E virus in Iran: a systematic review and meta-analysis. Middle East J Dig Dis. 2016;8(3):189-200. doi: 10.15171/ mejdd.2016.31.

22. Rostamzadeh Khameneh Z, Sepehrvand N, Masudi S, Seroprevalence of hepatitis $\mathrm{E}$ among Iranian renal transplant recipients. Hepat Mon. 2011;11(8):646-51.

23. Zeraati AA, Nazemian F, Takalloo L, Sahebkar A, Heidari E, Yaghoubi MA. The relationship between serology of hepatitis $\mathrm{E}$ virus with liver and kidney function in kidney transplant patients. Excli J. 2016;15:343-9 doi: 10.17179/excli2016-232.

24. Kamar N, Bendall R, Legrand-Abravanel F, Xia NS, ljaz S, Izopet J, et al. Hepatitis E. Lancet. 2012;379(9835):2477-88. doi: 10.1016/s0140-6736(11)61849-7. 